\title{
Review
}

Journal of Innate

Immunity
J Innate Immun 2012;4:241-247

DOI: $\underline{10.1159 / 000335029}$
Received: September 20, 2011

Accepted after revision: November 13, 2011

Published online: February 10, 2012

\section{Antimicrobial RNases in Cutaneous Defense}

\author{
Maren Simanski Bente Köten Jens-Michael Schröder Regine Gläser \\ Jürgen Harder \\ Department of Dermatology, University Hospital Schleswig-Holstein, Kiel Campus, Kiel, Germany
}

\section{Key Words \\ Antimicrobial proteins $\cdot$ Antimicrobial peptides . \\ Antimicrobial ribonucleases $\cdot$ Cutaneous defense}

\begin{abstract}
Antimicrobial proteins (AMP) are small endogenous proteins which are capable of rapidly inactivating microorganisms at low micro- and nanomolar concentrations. Their significance in host defense is reflected by their wide distribution in nature. Several AMP have been isolated from human skin, and there is increasing evidence that AMP may play an important role in cutaneous defense. One important human AMP class comprises several antimicrobial members of the RNase A superfamily. Of these, two members, RNase 7 and RNase 5, have been implicated in cutaneous defense. This review gives an overview about our current knowledge on the potential role of RNase 7 and RNase 5 in protecting human skin from infection.

Copyright $\odot 2012$ S. Karger AG, Basel
\end{abstract}

\section{Introduction}

Antimicrobial peptides and proteins (AMP) are endogenous antibiotic mediators which are able to rapidly kill a wide diversity of microorganisms. The importance of AMP in host defense is reflected by the wide distribution of these defense molecules in nature [1] and it seems obvious to speculate that AMP can be found in all higher organisms, including plants. Organisms without a sophisticated adaptive immunity and without professional phagocytic cells especially rely on the activity of AMP to control the outgrowth of invading microbes. Thus, it is not surprising that even simple metazoa such as the fresh water polyp Hydra uses AMP to control bacterial growth $[2,3]$. In addition, AMP are also produced by unicellular organisms such as amoebae and even bacteria are able to produce AMP to kill competing microorganisms [4, 5].

Given the obvious importance of AMP in host defense it seems logical that humans are also endowed with a diverse arsenal of AMP and consequently several different AMP classes have been identified in humans $[6,7]$. These AMP support innate and adaptive immunity through their antimicrobial and immunomodulatory activities (e.g. leukocyte chemotactic activities). In phagocytic cells such as neutrophil granulocytes, AMP contribute to the killing of ingested as well as extracellularly trapped microorganisms [8]. In addition, there is increasing evidence that AMP have a major function in epithelial defense to fight off potential pathogenic bacteria and to control the commensal flora $[9,10]$. The production of AMP by epithelial cells may explain the unexpected observation that epithelia are normally not infected despite

\section{KARGER}

Fax +4161306 1234

E-Mail karger@karger.ch

www.karger.com
(C) 2012 S. Karger AG, Basel

1662-811X/12/0043-0241\$38.00/0

Accessible online at:

www.karger.com/jin
Prof. Dr. Jürgen Harder

Department of Dermatology, University Hospital Schleswig-Holstein, Kiel Campus Schittenhelmstrasse 7

DE-24105 Kiel (Germany)

Tel. +49 431597 1598, E-Mail jharder@ dermatology.uni-kiel.de 
their presence and contact with a huge variety of microorganisms including commensals and potential pathogenic microbes.

Skin is a rich source of AMP and several human epithelial AMP have been isolated from skin extracts [11, 12]. Keratinocytes produce and release various AMP such as human $\beta$-defensins, S100 proteins, protease inhibitors and the cathelicidin LL-37 [13, 14]. Another important family of human AMP comprises members of the ribonuclease A superfamily. This family comprises several members expressing antimicrobial activity and two of them, RNase 7 and RNase 5, are subject of this review regarding their potential role in cutaneous defense.

\section{Antimicrobial Members of the Human RNase A Superfamily}

The name RNase A superfamily is based on bovine pancreatic RNase, also called RNase A. Members of the RNase A superfamily share sequence and structural similarities with RNase A such as 6-8 conserved cysteine residues forming characteristic disulfide bridges as well as conserved histidines and a lysine in the active center catalyzing the ribonuclease activity [15]. An amino acid alignment of human members of the RNase A superfamily is shown in figure 1 . Thirteen human genes encoding for RNase 1-13 have been identified on chromosome 14q11.2 [16]. Besides ribonucleolytic activity, biological activities such as neurotoxicity, angiogenic activity and immunomodulatory activity have been reported, but the physiological role of these RNases is still emerging [15].

Several members of the human RNase A superfamily have been associated with antimicrobial properties supporting the hypothesis that they may play a role in host defense. The first reports about a potential role of RNases in host defense came from studies about the human ribonucleases RNase 2 (eosinophil-derived neurotoxin) and RNase 3 (eosinophil cationic protein, ECP), which are both granule proteins of eosinophil granulocytes. Whereas eosinophil-derived neurotoxin has been associated only with antiviral activity, several studies have reported bactericidal, antiviral and antiparasitic activity of ECP $[17,18]$.

RNase 8 has been identified by searching the human genome databank and Northern blot analysis of 12 tissues identified RNase 8 gene expression only in placenta [19]. Using recombinant material we could show that RNase 8

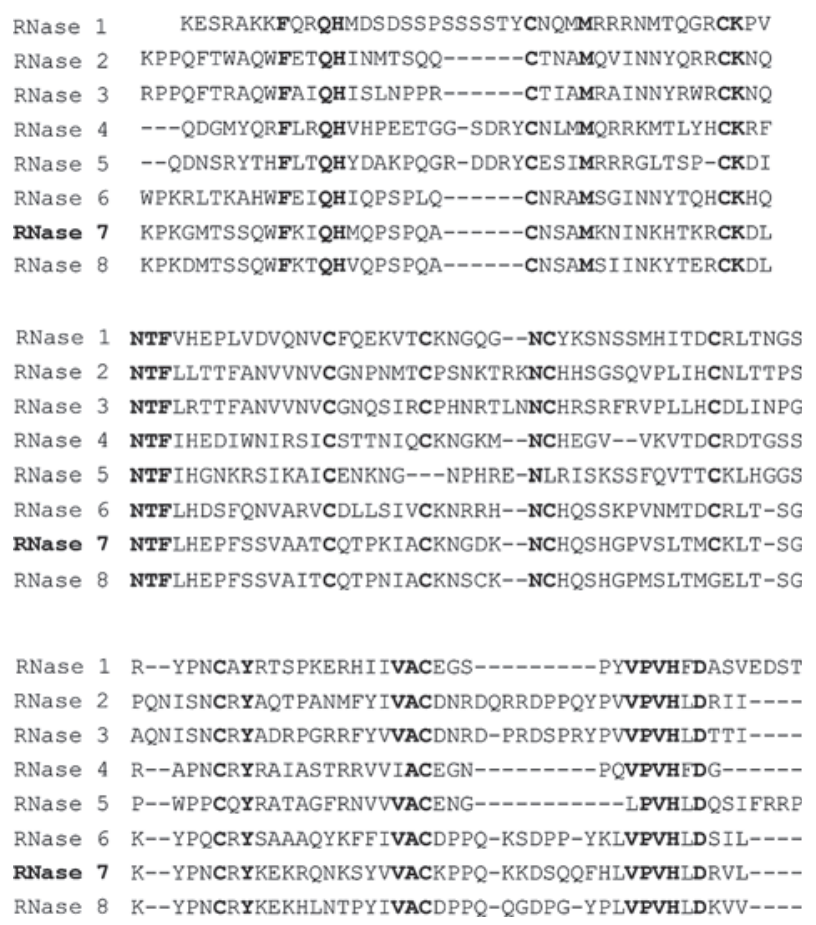

RNase 1 NTFVHEPLVDVQNVCFQEKVTCKNGQG--NCYKSNSSMHITDCRLTNGS RNase 2 NTFLLTTFANVVNVCGNPNMTCPSNKTRKNCHHSGSQVPLIHCNLTTPS RNase 3 NTFLRTTFANVVNVCGNQSIRCPHNRTLNNCHRSRFRVPLLHCDLINPG RNase 4 NTFIHEDIWNIRSICSTTNIQCKNGKM--NCHEGV--VKVTDCRDTGSS RNase 5 NTFIHGNKRSIKAICENKNG---NPHRE-NLRISKSSFQVTTCKLHGGS RNase 6 NTFLHDSFQNVARVCDLLSIVCKNRRH--NCHQSSKPVNMTDCRLT-SG RNase 7 NTFLHEPFSSVAATCQTPKIACKNGDK--NCHQSHGPVSLTMCKLT-SG RNaSe 8 NTFLHEPFSSVAITCQTPNIACKNSCK--NCHQSHGPMSLTMGELT-SG

RNase 1 R--YPNCAYRTSPKERHIIVACEGS---------PYVPVHFDASVEDST RNase 2 PQNISNCRYAQTPANMFYIVACDNRDQRRDPPQYPVVPVHLDRII---RNase 3 AQNISNCRYADRPGRRFYVVACDNRD-PRDSPRYPVVPVHLDTTI---RNase 4 R--APNCRYRAIASTRRVVIACEGN---------PQVPVHFDG-----RNase 5 P--WPPCQYRATAGFRNVVVACENG-----------LPVHLDQSIFRRE RNase 6 K--YPQCRYSAAAQYKFFIVACDPPQ-KSDPP-YKLVPVHLDSIL---RNase 7 K--YPNCRYKEKRQNKSYVVACKPPQ-KKDSQQFHLVPVHLDRVL---RNase 8 K--YPNCRYKEKHLNTPYIVACDPPQ-QGDPG-YPLVPVHLDKVV----

Fig. 1. Amino acid alignment of human members of the RNase A superfamily. The single-letter amino acid sequence of RNase 1 to RNase 8 is shown. Conserved amino acids are in bold. (This figure was originally published in Harder and Schröder [22]; ${ }^{\circ}$ the American Society for Biochemistry and Molecular Biology.)

exhibits potent bactericidal activity in vitro against a broad spectrum of bacteria [20]. Our own unpublished analysis of RNase 8 gene expression in keratinocytes revealed only very low transcript levels at the detection limit of the real-time PCR, suggesting that RNase 8 may play no role in cutaneous defense.

Like RNase 8, the native protein of RNase 9 has not yet been isolated. However, recombinant RNase 9 has been shown to exhibit antimicrobial activity against Escherichia coli. Since RNase 9 seems to be predominantly expressed in the epididymis, its antimicrobial activity suggests a potential function in host defense of the male reproductive tract [21].

RNase 5 and RNase 7 are two further antimicrobial members of the RNase A superfamily. Antimicrobial activity as well as expression in keratinocytes suggests that they participate in cutaneous defense. A detailed review of their antimicrobial characteristics and their potential role in skin defense is presented below. 


\section{RNase 7}

During analysis of skin-derived stratum corneum extracts for the presence of AMP we isolated a novel AMP which we termed RNase 7 [22]. At the same time Zhang et al. [23] identified RNase 7 through screening the human genome database. RNase 7 is a $14.5-\mathrm{kDa}$ cationic AMP with distinct ribonuclease activity. Natural skinderived RNase 7 exhibits potent in vitro antimicrobial activity against various Gram-negative and Gram-positive bacteria such as E. coli, Pseudomonas aeruginosa, Staphylococcus aureus, Enterococcus faecium, Propionibacterium acnes and against the yeast Candida albicans $[22,24]$. Notably, it is also active against multiresistant bacteria such as MRSA and vancomycin-resistant $E$. faecium [22].

Since RNase 7 is able to degrade RNA the question arises whether its antimicrobial activity depends on its ribonuclease activity. Huang et al. [25] generated a ribonuclease-inactive recombinant RNase 7 through mutation of catalytic histidines and lysine. This ribonucleasedeficient mutant exhibited similar activity against $P$. aeruginosa as the wild-type suggesting that the activity of RNase 7 against $P$. aeruginosa requires no functional ribonuclease activity. In line with this observation we found no significant differences in killing activities compared to the wild-type when testing a recombinant ribonuclease-deficient mutant against E. faecium and E. coli [24; unpubl. data]. Similar results have also been reported for ECP. Recombinant ribonuclease-deficient ECP was similarly active against $S$. aureus as the corresponding wild type [26]. It has also been reported that RNase A-2 from the chicken Gallus gallus exhibited ribonucleaseindependent activity against E. coli [27]. Together, all these data indicate that the antibacterial activity of RNase 7 functions independently of the ribonuclease activity. However, it is an interesting question as to whether the enzymatic activity is in general indispensable for antimicrobial activity or whether the killing of individual microorganisms requires intact ribonuclease activity. In this regard it has been shown that the ribonuclease activity of eosinophil-derived neurotoxin is essential for its activity against respiratory syncytial virus [28]. The strong ribonuclease activity of RNase 7 suggests that it also might have ribonuclease-dependent antiviral activity, a hypothesis which remains to be investigated. Induction of RNase 7 gene expression upon infection of keratinocytes with dengue virus supports this hypothesis [29].

The mechanism of the exact bactericidal action of RNase 7 is still emerging. First reports indicate that
RNase 7 binds and permeabilizes the bacterial membrane $[25,30]$. Membrane permeabilization requires four clustered lysine residues but no catalytic residues [25]. RNase 7 is able to bind to LPS with similar affinity as the known LPS-binding peptide polymyxin B. It also binds to peptidoglycan, the main component of the cell wall of Grampositive bacteria [31]. Scanning electron micrographs of E. coli and S. aureus treated with RNase 7 revealed the presence of local blebs on the bacterial cell surface and leakage assays demonstrated bacterial lysis upon RNase 7 treatment already after 1 and $2 \mathrm{~h}$ incubation of E. coli and $S$. aureus, respectively [31]. Lin et al. [32] reported that the binding of RNase 7 to the $P$. aeruginosa outer membrane protein I (OprI) is a prerequisite for its bactericidal action on $P$. aeruginosa. The activity of RNase 7 against $P$. aeruginosa was blocked by the addition of exogenous OprI or an anti-OprI antibody. These results indicate that the capacity to bind bacterial cell surface structures is a critical step in the bactericidal action of RNase 7. Further studies are necessary to decipher the exact bactericidal mechanism(s) of RNase 7 and to assess strain-dependent differences.

As mentioned above, RNase 7 has been originally isolated from stratum corneum skin extracts and an analysis of various tissues for RNase 7 transcript levels revealed the highest expression in skin, thus suggesting an important role of RNase 7 in cutaneous defense [22]. In addition, RNase 7 gene expression has also been detected in various other tissues, examples being the pharynx, tonsils and kidney. The functional relevance of RNase 7 in the kidney and the urinary tract has recently been shown through strong expression of RNase 7 detected in kidney and bladder tissue. Also, the antimicrobial activity of urine was decreased by addition of an RNase 7 neutralizing antibody [33].

In skin, the highest expression of RNase 7 can be detected in the stratum corneum as shown by immunohistochemistry [24]. The highest RNase 7 expression in the outermost, more differentiated epidermal layers is in concordance with induced RNase 7 expression in cultured differentiated keratinocytes [34]. A real-time PCR analysis showed that primary keratinocytes expressed high transcript levels of RNase 7 when compared to other skinderived AMP such as human $\beta$-defensin-2, psoriasin (S100 A7) and LL-37 [24]. In addition, an RT-PCR analysis of RNase 1 to RNase 8 gene expression in primary keratinocytes revealed expression of RNase 1, 4, 5 and 7, with the latter being the highest expressed [34]. ELISA analysis of cultured keratinocytes detected the majority of RNase 7 in the culture supernatants indicating that RNase 7 is 
Fig. 2. In vivo secretion of RNase 7 on the skin surface. Standardized skin areas were rinsed with sodium phosphate buffer and the concentrations of RNase 7 in the washing fluids were determined by an RNase 7 ELISA. The concentrations of one healthy volunteer are shown.
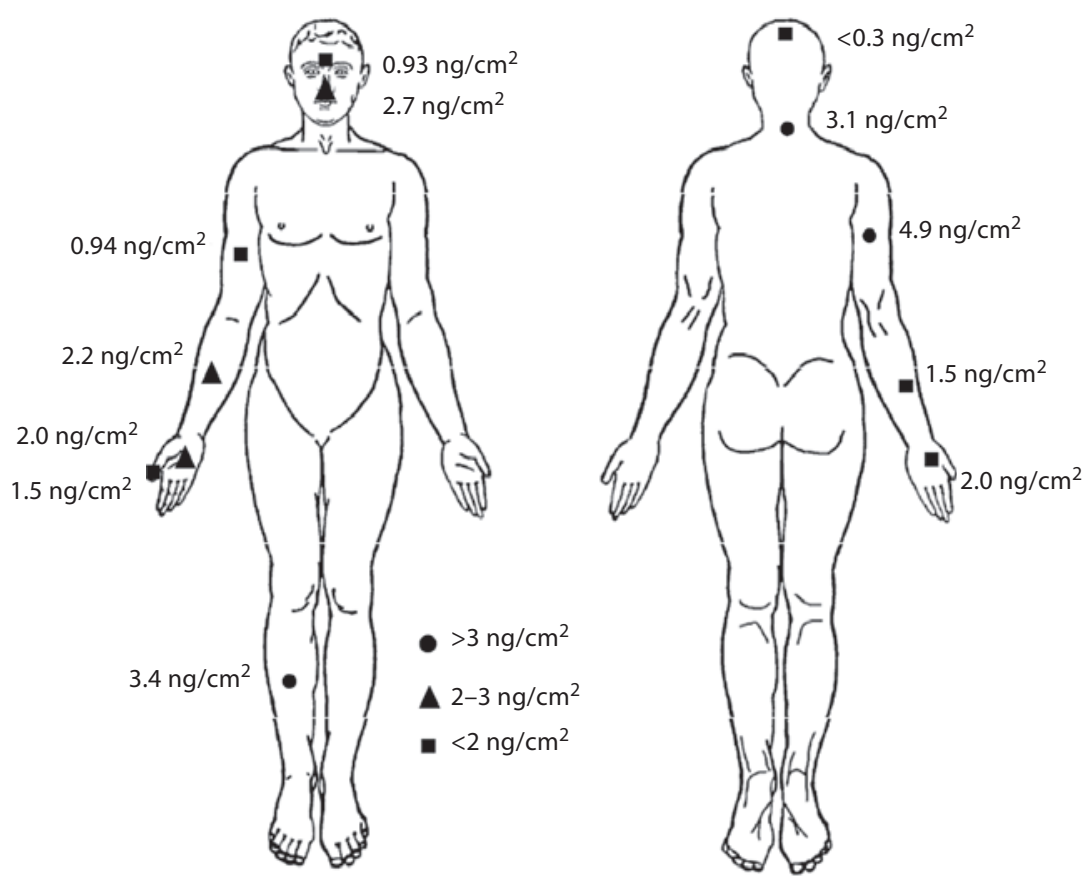

secreted and functions primarily outside the cell, which is in concordance with its proposed role in cutaneous defense [24]. In line with these observations, ELISA analysis of washing fluids obtained from various human skin locations revealed the presence of RNase 7 [24] (fig. 2). Expression of RNase 7 has also been reported in hair follicle epithelium suggesting a role of RNase 7 in protecting the hair follicle against microbial invasion [35].

The abundance of RNase 7 in the stratum corneum and in skin washing fluids suggests that it contributes to control of the growth of microorganisms on the skin surface. Corresponding to this hypothesis we could recently demonstrate the functional relevance of RNase 7 in controlling the growth of microorganisms on the skin surface. Incubation of stratum corneum extracts with an RNase 7-neutralizing antibody reduced the killing activity of the stratum corneum extracts against E. faecium [24]. Furthermore, application of an RNase 7-neutralizing antibody to the surface of skin explants enhanced the growth of applied S. aureus [36]. An important role of RNase 7 in protecting human skin from $S$. aureus infection has been further strengthened by an interesting observation made by Zanger et al. [37]. They found a significantly reduced RNase 7 gene expression in the unaffected skin of patients with $S$. aureus-positive skin infections.

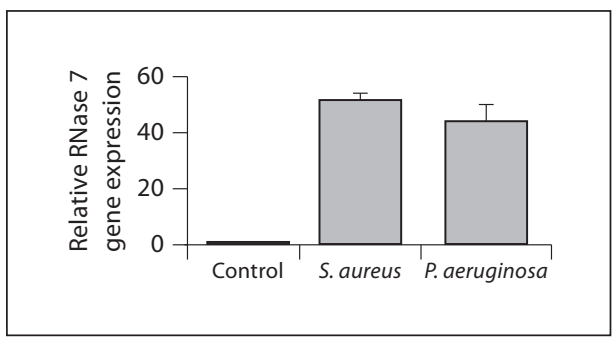

Fig. 3. Induction of RNase 7 gene expression in keratinocytes by bacteria. Primary keratinocytes were stimulated for $16 \mathrm{~h}$ with culture supernatants of $S$. aureus and $P$. aeruginosa. The relative RNase 7 gene expression normalized to the expression of the housekeeping gene GAPDH analyzed by real-time PCR is shown.

Despite the relatively high baseline levels of RNase 7 in keratinocytes, its expression can be further induced. Stimuli for the upregulation of RNase 7 in keratinocytes are proinflammatory cytokines such as interleukin- $1 \beta$ $[22,38]$, ultraviolet-B radiation [39] and bacteria such as $P$. aeruginosa [22] and S. aureus $[36,40]$ (fig. 3). Induction of RNase 7 was also seen in gingival epithelial cells simulated with biofilms of bacterial members of the oral flora $[41,42]$. 
The inducibility of RNase 7 might be the reason for the increased expression seen in the chronic inflammatory skin disease psoriasis. We isolated higher amounts of RNase 7 from psoriatic scales compared to the stratum corneum of healthy donors [11]. In addition, immunohistochemistry as well as ELISA analysis of RNase 7 amounts present on the lesional and nonlesional psoriatic skin surface revealed an association of increased RNase 7 expression with psoriasis [43]. This is in line with other skinderived AMP such as human $\beta$-defensin- 2 and psoriasin, which are also upregulated in psoriatic skin $[43,44]$. The increased expression of AMP in psoriasis may explain why cutaneous infections in psoriasis are unexpectedly rare despite the disturbed skin surface in psoriatic skin lesions [45].

Atopic dermatitis is another chronic inflammatory skin disease where AMP have been extensively studied. Several publications found decreased expression of some AMP in atopic dermatitis skin as compared to psoriasis, suggesting that there is an expression defect of AMP in atopic dermatitis. This might be responsible for the increased susceptibility of atopic dermatitis patients to $S$. aureus infection $[46,47]$. Subsequent studies revealed induced levels of AMP in atopic dermatitis, which were less pronounced when compared to psoriasis. However, by systematic analyses of atopic dermatitis skin with ageand sex-matched healthy skin samples from the same locations, a strong upregulation of gene and protein expression of several AMP including RNase 7 could be detected [43, 44]. Gambichler et al. [48] reported even higher RNase 7 gene expression in biopsies obtained from atopic dermatitis as compared to psoriasis. In addition, we were not able to detect a significant correlation of AMP expression and $S$. aureus colonization or disease severity of atopic dermatitis [43]. These data indicate that atopic dermatitis is not associated with a general induction defect of AMP. Clearly, further studies on the exact role of RNase 7 and other AMP in atopic dermatitis are needed.

\section{RNase 5}

RNase 5 was first named angiogenin because it was initially isolated as an angiogenic factor capable of inducing extensive blood vessel growth at low concentrations $[49,50]$. Characterization of its amino acid sequence and its structure identified angiogenin as a member of the human RNase A superfamily. Compared to RNase 7, RNase 5 displays less ribonucleolytic activity, which is still needed for full angiogenic activity [49].
Hooper et al. [51] reported that low micromolar concentrations of human RNase 5 exhibit antimicrobial activity against Streptococcus pneumoniae and the yeast $C$. albicans, while being ineffective against $E$. faecalis and Listeria monocytogenes. Contrasting results came from Avdeeva et al. [52] who reported a rather limited antimicrobial activity of RNase 5 against E. faecalis and L. monocytogenes, which was not higher than effects observed with bovine serum albumin alone. It is not clear whether differences in the used material are responsible for the contrasting data. Abtin et al. [34] could confirm activity of RNase 5 against C. albicans, but no activity was detected against the bacteria $P$. aeruginosa, E. coli, S. aureus (MRSA) or E. faecium. A potential explanation as to why RNase 5 does not have such a broad spectrum of antimicrobial activity as reported for RNase 7 could be the lack of several lysine residues required for membrane permeabilization of RNase 7 [25,34]. Treatment of RNase 5 with DEPC and benzopurpurin, which both block the ribonucleolytic activity of RNase 5, led to a strong reduction of the candidacidal activity of RNase 5. This indicates that the enzymatic activity is a prerequisite for its capacity to kill C. albicans, although further studies using point-mutated variants of recombinant RNase 5 are required to verify this hypothesis [34].

As mentioned above, a PCR-based gene expression analysis revealed expression of RNase 5 in primary keratinocytes [34]. As shown for RNase 7, expression of RNase 5 was also induced in differentiated keratinocytes. This suggests an expression of RNase 5 in the more differentiated outermost epidermal layers which has to be proven by immunohistochemistry. Nevertheless, it has been shown that RNase 5 can be detected at the surface of in vitro reconstructed epidermis indicating the capacity of human skin to express and secrete RNase 5 [53]. Cutaneous expression of RNase 5 may point towards a role in protecting skin against infection, although the functional relevance of RNase 5 in controlling microbial growth at the skin surface needs to be demonstrated.

\section{Interaction with the Ribonuclease Inhibitor}

It has been shown that the antimicrobial activity of RNase 5 and RNase 7 is inhibited when incubated with the ribonuclease inhibitor [34]. Although structural changes rather than a specific inhibition of ribonucleolytic activity of these RNases might be responsible for the decreased antimicrobial activity, the binding of the ribonuclease inhibitor could regulate antimicrobial activities

J Innate Immun 2012;4:241-247 
of RNases in vivo. RNase 5 and RNase 7 are both present in the stratum corneum, whereas the ribonuclease inhibitor is absent in the stratum corneum but present in deeper epidermal layers. The reason might be that the stratum corneum contains proteolytic activity which is able to degrade the ribonuclease inhibitor [34]. This led to the hypothesis that degradation of the ribonuclease inhibitor in the stratum corneum abrogates the ribonuclease inhibitor-mediated inhibition of RNase 5 and RNase 7 leading to activation of the antimicrobial activity of these two RNases [34, 54].

\section{Conclusion}

As discussed in this review, the existing data make it likely that RNases may play an important role in cutaneous defense. This hypothesis is supported by functional studies of RNase 7 using neutralizing antibodies as well as demonstration of an association of increased susceptibility to cutaneous $S$. aureus infections with lower RNase 7 expression. Clearly, further studies are needed to assess the role of antimicrobial RNases in the skin barrier. Uncovering the physiological function of the ribonucleolytic activity associated with antimicrobial RNases is a further important goal.

\section{Acknowledgments}

Parts of the work presented herein were supported by grants from the German Research Foundation (DFG) to J.H. and J.-M.S. (HA 3386/3-1, HA 3386/6-1, SFB 617) and the Federal Ministry of Education and Research (SkinStaph to R.G. and J.H.).

\section{Disclosure Statement}

The authors state no conflict of interest.

\section{References}

1 Harder J: Antimicrobial peptides: ancient molecules as modern therapeutics? Expert Rev Dermatol 2008;3:1-5.

- 2 Bosch TC, Augustin R, Anton-Erxleben F, Fraune S, Hemmrich G, Zill H, Rosenstiel P, Jacobs G, Schreiber S, Leippe M, Stanisak M, Grotzinger J, Jung S, Podschun R, Bartels J, Harder J, Schröder JM: Uncovering the evolutionary history of innate immunity: the simple metazoan hydra uses epithelial cells for host defence. Dev Comp Immunol 2009; 33:559-569.

- 3 Fraune S, Augustin R, Anton-Erxleben F, Wittlieb J, Gelhaus C, Klimovich VB, Samoilovich MP, Bosch TC: In an early branching metazoan, bacterial colonization of the embryo is controlled by maternal antimicrobial peptides. Proc Natl Acad Sci USA 2010;107:18067-18072.

-4 Leippe M, Herbst R: Ancient weapons for attack and defense: the pore-forming polypeptides of pathogenic enteric and free-living amoeboid protozoa. J Eukaryot Microbiol 2004;51:516-521.

5 Sahl HG: Gene-encoded antibiotics made in bacteria. Ciba Found Symp 1994;186:27-53.

6 Harder J, Gläser R, Schröder JM: Human antimicrobial proteins effectors of innate immunity. J Endotoxin Res 2007;13:317-338.

7 Wiesner J, Vilcinskas A: Antimicrobial peptides: The ancient arm of the human immune system. Virulence 2010;1:440-464.
8 Soehnlein O: Direct and alternative antimicrobial mechanisms of neutrophil-derived granule proteins. J Mol Med (Berl) 2009;87: 1157-1164

-9 Bevins CL, Salzman NH: Paneth cells, antimicrobial peptides and maintenance of intestinal homeostasis. Nat Rev Microbiol 2011;9:356-368.

10 Harder J, Gläser R, Schröder JM: The role and potential therapeutical applications of antimicrobial proteins in infectious and inflammatory diseases. Endocr Metab Immune Disord Drug Targets 2007;7:75-82.

11 Harder J, Schröder JM: Psoriatic scales: a promising source for the isolation of human skin-derived antimicrobial proteins. J Leukoc Biol 2005;77:476-486.

12 Schröder JM, Harder J: Antimicrobial skin peptides and proteins. Cell Mol Life Sci 2006; 63:469-486.

13 Harder J, Schröder JM: Antimicrobial peptides in human skin. Chem Immunol Allergy 2005;86:22-41.

14 Schauber J, Gallo RL: Antimicrobial peptides and the skin immune defense system. J Allergy Clin Immunol 2009;124:R13-R18.

15 Sorrentino S: The eight human 'canonical' ribonucleases: molecular diversity, catalytic properties, and special biological actions of the enzyme proteins. FEBS Lett 2010;584: 2194-2200.

16 Cho S, Beintema JJ, Zhang J: The ribonuclease A superfamily of mammals and birds: identifying new members and tracing evolutionary histories. Genomics 2005;85:208-220.
17 Rosenberg HF: RNase A ribonucleases and host defense: an evolving story. J Leukoc Biol 2008;83:1079-1087.

18 Boix E, Nogues MV: Mammalian antimicrobial proteins and peptides: overview on the RNase A superfamily members involved in innate host defence. Mol Biosyst 2007;3:317335.

19 Zhang J, Dyer KD, Rosenberg HF: RNase 8, a novel RNase A superfamily ribonuclease expressed uniquely in placenta. Nucleic Acids Res 2002;30:1169-1175.

20 Rudolph B, Podschun R, Sahly H, Schubert S, Schröder JM, Harder J: Identification of RNase 8 as a novel human antimicrobial protein. Antimicrob Agents Chemother 2006; 50:3194-3196.

21 Cheng GZ, Li JY, Li F, Wang HY, Shi GX: Human ribonuclease 9, a member of ribonuclease A superfamily, specifically expressed in epididymis, is a novel sperm-binding protein. Asian J Androl 2009;11:240-251.

22 Harder J, Schröder JM: RNase 7, a novel innate immune defense antimicrobial protein of healthy human skin. J Biol Chem 2002; 277:46779-46784.

23 Zhang J, Dyer KD, Rosenberg HF: Human RNase 7: a new cationic ribonuclease of the RNase A superfamily. Nucleic Acids Res 2003;31:602-607.

-24 Köten B, Simanski M, Gläser R, Podschun R, Schröder JM, Harder J: RNase 7 contributes to the cutaneous defense against Enterococcus faecium. PLoS One 2009;4:e6424. 
-25 Huang YC, Lin YM, Chang TW, Wu SJ, Lee YS, Chang MD, Chen C, Wu SH, Liao YD: The flexible and clustered lysine residues of human ribonuclease 7 are critical for membrane permeability and antimicrobial activity. J Biol Chem 2007;282:4626-4633.

-26 Venge P, Bystrom J, Carlson M, Hakansson L, Karawacjzyk M, Peterson C, Seveus L, Trulson A: Eosinophil cationic protein (ECP): molecular and biological properties and the use of ECP as a marker of eosinophil activation in disease. Clin Exp Allergy 1999; 29:1172-1186.

-27 Nitto T, Dyer KD, Czapiga M, Rosenberg HF: Evolution and function of leukocyte RNase A ribonucleases of the avian species, Gallus gallus. J Biol Chem 2006;281:25622-25634.

-28 Domachowske JB, Dyer KD, Bonville CA, Rosenberg HF: Recombinant human eosinophil-derived neurotoxin/RNase 2 functions as an effective antiviral agent against respiratory syncytial virus. J Infect Dis 1998;177: 1458-1464.

-29 Surasombatpattana P, Hamel R, Patramool S, Luplertlop N, Thomas F, Despres P, Briant L, Yssel H, Misse D: Dengue virus replication in infected human keratinocytes leads to activation of antiviral innate immune responses. Infect Genet Evol 2011;11:1664-1673.

-30 Torrent M, Sanchez D, Buzon V, Nogues MV, Cladera J, Boix E: Comparison of the membrane interaction mechanism of two antimicrobial RNases: RNase 3/ECP and RNase 7. Biochim Biophys Acta 2009; 1788:1116-1125.

- 31 Torrent M, Badia M, Moussaoui M, Sanchez D, Nogues MV, Boix E: Comparison of human RNase 3 and RNase 7 bactericidal action at the gram-negative and gram-positive bacterial cell wall. FEBS J 2010;277:17131725.

-32 Lin YM, Wu SJ, Chang TW, Wang CF, Suen CS, Hwang MJ, Chang MD, Chen YT, Liao YD: Outer membrane protein I of Pseudomonas aeruginosa is a target of cationic antimicrobial peptide/protein. J Biol Chem 2010; 285:8985-8994.

- 33 Spencer JD, Schwaderer AL, Dirosario JD, McHugh KM, McGillivary G, Justice SS, Carpenter AR, Baker PB, Harder J, Hains DS: Ribonuclease 7 is a potent antimicrobial peptide within the human urinary tract. Kidney Int 2011;80:174-180.
4 Abtin A, Eckhart L, Mildner M, Ghannadan M, Harder J, Schröder JM, Tschachler E: Degradation by stratum corneum proteases prevents endogenous RNase inhibitor from blocking antimicrobial activities of RNase 5 and RNase 7. J Invest Dermatol 2009;129: 2193-2201.

35 Reithmayer K, Meyer KC, Kleditzsch P, Tiede S, Uppalapati SK, Gläser R, Harder J, Schröder JM, Paus R: Human hair follicle epithelium has an antimicrobial defence system that includes the inducible antimicrobial peptide psoriasin (s100a7) and RNase 7. Br J Dermatol 2009;161:78-89.

-36 Simanski M, Dressel S, Gläser R, Harder J: RNase 7 protects healthy skin from Staphylococcus aureus colonization. J Invest Dermatol 2010;130:2836-2838.

37 Zanger P, Holzer J, Schleucher R, Steffen H, Schittek B, Gabrysch S: Constitutive expression of the antimicrobial peptide RNase 7 is associated with Staphylococcus aureus infection of the skin. J Infect Dis 2009;200:19071915.

38 Mohammed I, Yeung A, Abedin A, Hopkinson A, Dua HS: Signalling pathways involved in ribonuclease-7 expression. Cell Mol Life Sci 2011;68:1941-1952.

-39 Gläser R, Navid F, Schuller W, Jantschitsch C, Harder J, Schröder JM, Schwarz A, Schwarz T: UV-B radiation induces the expression of antimicrobial peptides in human keratinocytes in vitro and in vivo. J Allergy Clin Immunol 2009;123:1117-1123.

40 Wanke I, Steffen H, Christ C, Krismer B, Gotz F, Peschel A, Schaller M, Schittek B: Skin commensals amplify the innate immune response to pathogens by activation of distinct signaling pathways. J Invest Dermatol 2011;131:382-390.

41 Eberhard J, Pietschmann R, Falk W, Jepsen $\mathrm{S}$, Dommisch H: The immune response of oral epithelial cells induced by single-species and complex naturally formed biofilms. Oral Microbiol Immunol 2009;24:325-330.

-42 Eberhard J, Menzel N, Dommisch H, Winter J, Jepsen S, Mutters R: The stage of native biofilm formation determines the gene expression of human beta-defensin-2, psoriasin, ribonuclease 7 and inflammatory mediators: a novel approach for stimulation of keratinocytes with in situ formed biofilms. Oral Microbiol Immunol 2008;23:21-28.

-43 Harder J, Dressel S, Wittersheim M, Cordes J, Meyer-Hoffert U, Mrowietz U, FolsterHolst R, Proksch E, Schröder JM, Schwarz T, Gläser R: Enhanced expression and secretion of antimicrobial peptides in atopic dermatitis and after superficial skin injury. J Invest Dermatol 2010;130:1355-1364.
44 de Jongh GJ, Zeeuwen PL, Kucharekova M, Pfundt R, van der Valk PG, Blokx W, Dogan A, Hiemstra PS, van de Kerkhof PC, Schalkwijk J: High expression levels of keratinocyte antimicrobial proteins in psoriasis compared with atopic dermatitis. J Invest Dermatol 2005; 125:1163-1173.

45 Henseler T, Christophers E: Disease concomitance in psoriasis. J Am Acad Dermatol 1995;32:982-986.

46 Nomura I, Goleva E, Howell MD, Hamid QA, Ong PY, Hall CF, Darst MA, Gao B, Boguniewicz M, Travers JB, Leung DY: Cytokine milieu of atopic dermatitis, as compared to psoriasis, skin prevents induction of innate immune response genes. J Immunol 2003;171:3262-3269.

-47 Ong PY, Ohtake T, Brandt C, Strickland I, Boguniewicz M, Ganz T, Gallo RL, Leung DY: Endogenous antimicrobial peptides and skin infections in atopic dermatitis. $\mathrm{N}$ Engl J Med 2002;347:1151-1160.

-48 Gambichler T, Skrygan M, Tomi NS, Othlinghaus $\mathrm{N}$, Brockmeyer $\mathrm{NH}$, Altmeyer $\mathrm{P}$, Kreuter A: Differential mRNA expression of antimicrobial peptides and proteins in atopic dermatitis as compared to psoriasis vulgaris and healthy skin. Int Arch Allergy Immunol 2008;147:17-24.

49 Gao X, Xu Z: Mechanisms of action of angiogenin. Acta Biochim Biophys Sin (Shanghai) 2008;40:619-624.

50 Fett JW, Strydom DJ, Lobb RR, Alderman EM, Bethune JL, Riordan JF, Vallee BL: Isolation and characterization of angiogenin, an angiogenic protein from human carcinoma cells. Biochemistry 1985;24:5480-5486.

51 Hooper LV, Stappenbeck TS, Hong CV, Gordon JI: Angiogenins: a new class of microbicidal proteins involved in innate immunity. Nat Immunol 2003;4:269-273.

52 Avdeeva SV, Chernukha MU, Shaginyan IA, Tarantul VZ, Naroditsky BS: Human angiogenin lacks specific antimicrobial activity Curr Microbiol 2006;53:477-478.

53 Rendl M, Mayer C, Weninger W, Tschachler E: Topically applied lactic acid increases spontaneous secretion of vascular endothelial growth factor by human reconstructed epidermis. Br J Dermatol 2001;145:3-9.

54 Zasloff M: Antimicrobial RNases of human skin. J Invest Dermatol 2009;129:2091-2093. 